\title{
Variability of Uranyl Carboxylates from Rigid Terophenyl Dicarboxylic Acid Ligands
}

\author{
Xiaomin Hou and Si-Fu Tang* \\ ${ }^{\dagger}$ Shandong Province Key Laboratory of Applied Mycology, College of Life Science \\ and $\$$ College of Chemistry and Pharmaceutical Sciences, Qingdao Agricultural \\ University, Changcheng Road 700, Chengyang District, Qingdao 266109, China.
}

Instruments. The contents of $\mathrm{C}, \mathrm{H}$ and $\mathrm{N}$ were analyzed on an elemental analyzer (Vario EL III). Powder X-ray diffraction patterns (PXRD) were recorded on a Bruker D8 Advance diffractometer with $\mathrm{CuK} \alpha$ radiation (see Figure $\mathrm{S} 1-\mathrm{S} 3$ in the supplementary file). The IR spectra (see Figure S4) were measured on a Spectrometer (Thermo Fisher Nicolet iS-10) in the range of 4000-400 $\mathrm{cm}^{-1}$ using $\mathrm{KBr}$ pellets. Thermogravimetric analyses (TGA) were carried out on a NETZSCH STA 449C unit at a heating rate of $10{ }^{\circ} \mathrm{C} / \mathrm{min}$ under nitrogen atmosphere. Emission spectra of compounds 1-3 were recorded on a HITACHI F-7000 FL spectrophotometer. The UV-Vis spectra of ligands $\mathrm{H}_{2} \mathrm{~L}^{1}$ and $\mathrm{H}_{2} \mathrm{~L}^{2}$ and compounds 1-3 were measured on HITACHI U-3900 spectrophotometer.

Single-Crystal Structure Determination. The diffraction intensity data of compounds 1-3 were collected on a Bruker SMART APEX II CCD diffractometer (Mo K $\alpha$ radiation, $\lambda=0.71073 \AA$ ) at room temperature. The intensity of reflections and scaling were performed with $\mathrm{SAINT}^{1}$. Absorption corrections were performed using the program SADABS ${ }^{2}$. The crystal structures were solved by direct methods using SHELXS ${ }^{3}$. The atom positions were localized according to the difference Fourier analyses and least squares refinement using SHELXL-2013 ${ }^{4}$. Non-hydrogen atoms except $\mathrm{O} 1 \mathrm{w}$ in compound $\mathbf{1}$ and the acetonitrile molecule $(\mathrm{C} 23, \mathrm{C} 24, \mathrm{~N} 1)$ in compound $\mathbf{3}$ were refined with anisotropic thermal parameters. The hydrogen atoms on the water molecules were located from the difference Fourier map. All hydrogen atoms were refined using a riding model. Some guest molecules in the channels of compound 1 were disordered and could not be modeled, their electron density peaks were deleted using the SQUEEZE function implemented in PLATON ${ }^{5}$. The data have been deposited in the Cambridge Crystallographic Data Centre (CCDC), deposition numbers CCDC 2017180-2017182 for compounds 1-3. These data can be obtained free of charge via www.ccdc.cam.ac.uk/data_request/cif, or by emailing data_request@ccdc.cam.ac.uk, or by contacting The Cambridge Crystallographic Data Centre, 12, Union Road, Cambridge CB2 1EZ, UK; fax: +44 1223336033.

(1) SAINT, Version 6.45; Bruker Analytical X-ray Systems Inc.: 2003. 
(2) Sheldrick, G. M. SADABS, Version 2.10; Bruker AXS Inc.: Madison, WI, 2003.

(3) Sheldrick, G. M. SHELXS-97, Program for Crystal Structure Solution and Refinement; University of Göttingen, 1997.

(4) Sheldrick, G. M. SHELXL 2013 (Univ. Göttingen, 2013).

(5) Spek, A. L. Structure validation in chemical crystallography. Acta Cryst., 2009, D65, 148-155.

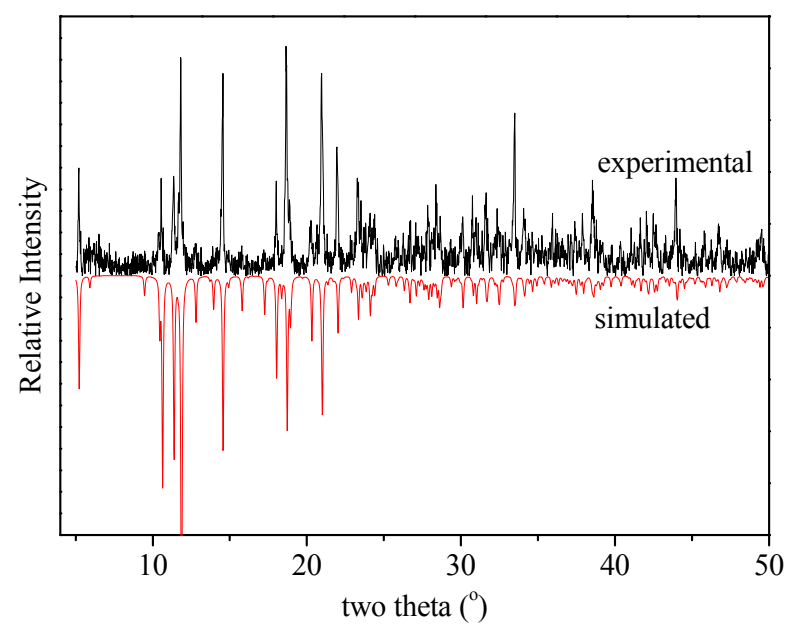

Figure S1. Powder XRD pattern of compound 1.

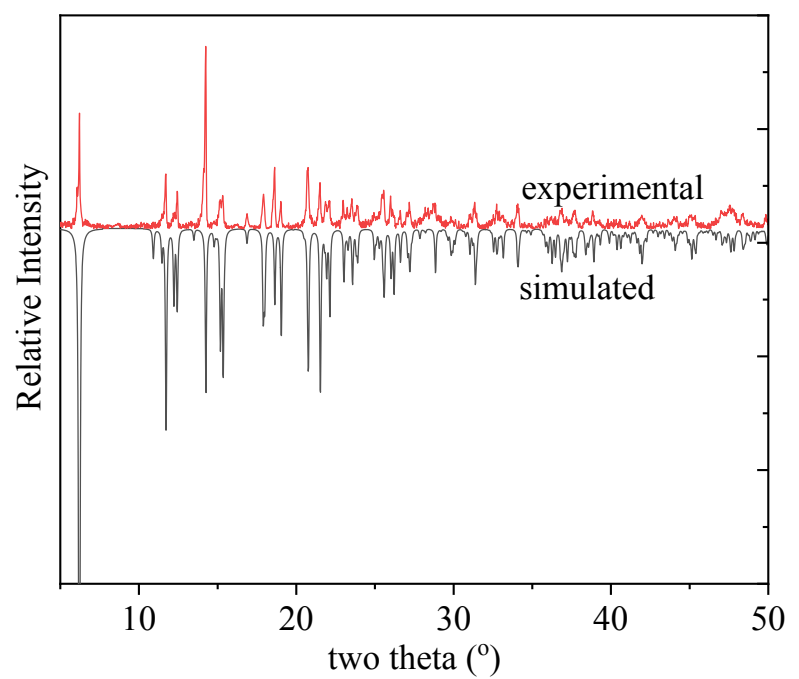

Figure S2. Powder XRD pattern of compound 2. 




Figure S3. Powder XRD pattern of compound 3.



Figure S4. IR spectra of compounds 1-3.

Table S1. Selected angles $\left(^{\circ}\right)$ in compounds 1-3.

\begin{tabular}{|l|l|l|l|}
\hline \multicolumn{5}{|l|}{ Compound 1 } & $178.3(5)$ & $\mathrm{O}(5)-\mathrm{U}(1)-\mathrm{O}(1) \# 2$ & $93.0(4)$ \\
\hline $\mathrm{O}(6)-\mathrm{U}(1)-\mathrm{O}(5)$ & $89.7(4)$ & $\mathrm{O}(4)-\mathrm{U}(1)-\mathrm{O}(1) \# 2$ & $129.8(2)$ \\
\hline $\mathrm{O}(6)-\mathrm{U}(1)-\mathrm{O}(4)$ & $88.8(5)$ & $\mathrm{O}(3) \# 1-\mathrm{U}(1)-\mathrm{O}(1) \# 2$ & $147.9(3)$ \\
\hline $\mathrm{O}(5)-\mathrm{U}(1)-\mathrm{O}(4)$ & $91.4(3)$ & $\mathrm{O}(1 \mathrm{~W})-\mathrm{U}(1)-\mathrm{O}(1) \# 2$ & $73.4(3)$ \\
\hline $\mathrm{O}(6)-\mathrm{U}(1)-\mathrm{O}(3) \# 1$ & $89.2(4)$ & $\mathrm{O}(6)-\mathrm{U}(1)-\mathrm{O}(2) \# 2$ & $89.3(3)$ \\
\hline $\mathrm{O}(5)-\mathrm{U}(1)-\mathrm{O}(3) \# 1$ & $82.2(3)$ & $\mathrm{O}(5)-\mathrm{U}(1)-\mathrm{O}(2) \# 2$ & $89.6(4)$ \\
\hline $\mathrm{O}(4)-\mathrm{U}(1)-\mathrm{O}(3) \# 1$ & $96.4(5)$ & $\mathrm{O}(4)-\mathrm{U}(1)-\mathrm{O}(2) \# 2$ & $77.1(2)$ \\
\hline $\mathrm{O}(6)-\mathrm{U}(1)-\mathrm{O}(1 \mathrm{~W})$ & $85.3(6)$ & $\mathrm{O}(3) \# 1-\mathrm{U}(1)-\mathrm{O}(2) \# 2$ & $159.4(3)$ \\
\hline $\mathrm{O}(5)-\mathrm{U}(1)-\mathrm{O}(1 \mathrm{~W})$ & $156.5(4)$ & $\mathrm{O}(1 \mathrm{~W})-\mathrm{U}(1)-\mathrm{O}(2) \# 2$ & $125.5(3)$ \\
\hline $\mathrm{O}(4)-\mathrm{U}(1)-\mathrm{O}(1 \mathrm{~W})$ & $74.9(4)$ & $\mathrm{O}(1) \# 2-\mathrm{U}(1)-\mathrm{O}(2) \# 2$ & $52.7(2)$ \\
\hline $\mathrm{O}(3) \# 1-\mathrm{U}(1)-\mathrm{O}(1 \mathrm{~W})$ & &
\end{tabular}




\begin{tabular}{|c|c|c|c|}
\hline $\mathrm{O}(6)-\mathrm{U}(1)-\mathrm{O}(1) \# 2$ & $87.4(4)$ & & \\
\hline \multicolumn{4}{|l|}{ Compound 2} \\
\hline $\mathrm{O}(6)-\mathrm{U}(1)-\mathrm{O}(5)$ & $179.0(2)$ & $\mathrm{O}(5)-\mathrm{U}(1)-\mathrm{O}(2) \# 2$ & $86.88(19)$ \\
\hline $\mathrm{O}(6)-\mathrm{U}(1)-\mathrm{O}(4)$ & $86.55(18)$ & $\mathrm{O}(4)-\mathrm{U}(1)-\mathrm{O}(2) \# 2$ & $129.99(15)$ \\
\hline $\mathrm{O}(5)-\mathrm{U}(1)-\mathrm{O}(4)$ & $92.53(19)$ & $\mathrm{O}(3) \# 1-\mathrm{U}(1)-\mathrm{O}(2) \# 2$ & $75.22(15)$ \\
\hline $\mathrm{O}(6)-\mathrm{U}(1)-\mathrm{O}(3) \# 1$ & $88.40(18)$ & $\mathrm{O}(1 \mathrm{~W})-\mathrm{U}(1)-\mathrm{O}(2) \# 2$ & $149.91(16)$ \\
\hline $\mathrm{O}(5)-\mathrm{U}(1)-\mathrm{O}(3) \# 1$ & $92.2(2)$ & $\mathrm{O}(6)-\mathrm{U}(1)-\mathrm{O}(1) \# 2$ & $91.09(19)$ \\
\hline $\mathrm{O}(4)-\mathrm{U}(1)-\mathrm{O}(3) \# 1$ & $154.57(16)$ & $\mathrm{O}(5)-\mathrm{U}(1)-\mathrm{O}(1) \# 2$ & $89.2(2)$ \\
\hline $\mathrm{O}(6)-\mathrm{U}(1)-\mathrm{O}(1 \mathrm{~W})$ & $95.36(18)$ & $\mathrm{O}(4)-\mathrm{U}(1)-\mathrm{O}(1) \# 2$ & $77.38(15)$ \\
\hline $\mathrm{O}(5)-\mathrm{U}(1)-\mathrm{O}(1 \mathrm{~W})$ & $83.96(19)$ & $\mathrm{O}(3) \# 1-\mathrm{U}(1)-\mathrm{O}(1) \# 2$ & $127.66(16)$ \\
\hline $\mathrm{O}(4)-\mathrm{U}(1)-\mathrm{O}(1 \mathrm{~W})$ & $79.12(16)$ & $\mathrm{O}(1 \mathrm{~W})-\mathrm{U}(1)-\mathrm{O}(1) \# 2$ & $155.19(17)$ \\
\hline $\mathrm{O}(3) \# 1-\mathrm{U}(1)-\mathrm{O}(1 \mathrm{~W})$ & $76.56(16)$ & $\mathrm{O}(2) \# 2-\mathrm{U}(1)-\mathrm{O}(1) \# 2$ & $52.61(14)$ \\
\hline $\mathrm{O}(6)-\mathrm{U}(1)-\mathrm{O}(2) \# 2$ & $94.10(19)$ & & \\
\hline \multicolumn{4}{|l|}{ Compound $\mathbf{3}$} \\
\hline $\mathrm{O}(5)-\mathrm{U}(1)-\mathrm{O}(6)$ & $178.8(4)$ & $\mathrm{O}(6)-\mathrm{U}(1)-\mathrm{O}(1) \# 2$ & $91.1(4)$ \\
\hline $\mathrm{O}(5)-\mathrm{U}(1)-\mathrm{O}(3) \# 1$ & $93.5(4)$ & $\mathrm{O}(3) \# 1-\mathrm{U}(1)-\mathrm{O}(1) \# 2$ & $128.6(3)$ \\
\hline $\mathrm{O}(6)-\mathrm{U}(1)-\mathrm{O}(3) \# 1$ & $87.1(3)$ & $\mathrm{O}(4)-\mathrm{U}(1)-\mathrm{O}(1) \# 2$ & $75.5(3)$ \\
\hline $\mathrm{O}(5)-\mathrm{U}(1)-\mathrm{O}(4)$ & $92.6(4)$ & $\mathrm{O}(1 \mathrm{~W})-\mathrm{U}(1)-\mathrm{O}(1) \# 2$ & $150.3(3)$ \\
\hline $\mathrm{O}(6)-\mathrm{U}(1)-\mathrm{O}(4)$ & $87.3(3)$ & $\mathrm{O}(5)-\mathrm{U}(1)-\mathrm{O}(2) \# 2$ & $87.0(4)$ \\
\hline $\mathrm{O}(3) \# 1-\mathrm{U}(1)-\mathrm{O}(4)$ & $155.3(3)$ & $\mathrm{O}(6)-\mathrm{U}(1)-\mathrm{O}(2) \# 2$ & $92.1(3)$ \\
\hline $\mathrm{O}(5)-\mathrm{U}(1)-\mathrm{O}(1 \mathrm{~W})$ & $84.7(4)$ & $\mathrm{O}(3) \# 1-\mathrm{U}(1)-\mathrm{O}(2) \# 2$ & $75.9(3)$ \\
\hline $\mathrm{O}(6)-\mathrm{U}(1)-\mathrm{O}(1 \mathrm{~W})$ & $96.5(3)$ & $\mathrm{O}(4)-\mathrm{U}(1)-\mathrm{O}(2) \# 2$ & $128.4(3)$ \\
\hline $\mathrm{O}(3) \# 1-\mathrm{U}(1)-\mathrm{O}(1 \mathrm{~W})$ & $80.6(3)$ & $\mathrm{O}(1 \mathrm{~W})-\mathrm{U}(1)-\mathrm{O}(2) \# 2$ & $154.5(3)$ \\
\hline $\mathrm{O}(4)-\mathrm{U}(1)-\mathrm{O}(1 \mathrm{~W})$ & $76.1(3)$ & $\mathrm{O}(1) \# 2-\mathrm{U}(1)-\mathrm{O}(2) \# 2$ & $52.9(3)$ \\
\hline $\mathrm{O}(5)-\mathrm{U}(1)-\mathrm{O}(1) \# 2$ & $87.7(4)$ & & \\
\hline
\end{tabular}

Symmetry transformations used to generate equivalent atoms: For 1: $\# 1-x, y,-z+3 / 2$; $\# 2-x+1 / 2, y+1 / 2, z+1$. For 2 : $\# 1-x+1, y+1 / 2,-z+3 / 2$; \#2 -x, $y+1 / 2,-z+1 / 2$. For 3: $\# 1$ $-\mathrm{x}+3, \mathrm{y}-1 / 2,-\mathrm{z}+5 / 2 ; \# 2 \mathrm{x}+1, \mathrm{y}, \mathrm{z}+1$.

Table S2. Analysis of Short Ring-Interactions with $\mathrm{Cg} \cdots \mathrm{Cg}$ Distances $<6.0$ Angstrom and Beta $<60.0$ Deg.

$\mathrm{Cg}(\mathrm{I})=$ Plane number $\mathrm{I}$ (= ring number in ( $)$ above)

Alpha $=$ Dihedral Angle between Planes I and J (Deg)

Beta $=$ Angle $\mathrm{Cg}(\mathrm{I})-->\mathrm{Cg}(\mathrm{J})$ or $\mathrm{Cg}(\mathrm{I})-->$ Me vector and normal to plane I (Deg)

Gamma $=$ Angle $\mathrm{Cg}(\mathrm{I})-->\mathrm{Cg}(\mathrm{J})$ vector and normal to plane $\mathrm{J}(\mathrm{Deg})$

$\mathrm{Cg} \cdots \mathrm{Cg}=$ Distance between ring Centroids (Ang.)

CgI_Perp $=$ Perpendicular distance of $\mathrm{Cg}(\mathrm{I})$ on ring $\mathrm{J}$ (Ang.)

CgJ_Perp $=$ Perpendicular distance of $\mathrm{Cg}(\mathrm{J})$ on ring I (Ang.)

For 1:

$\mathrm{Cg} 1=\mathrm{C}(2)-\mathrm{C}(3)-\mathrm{C}(4)-\mathrm{C}(5)-\mathrm{C}(6)-\mathrm{C}(7) \rightarrow$

$\mathrm{Cg} 2=\mathrm{C}(8)-\mathrm{C}(9)-\mathrm{C}(10)-\mathrm{C}(11)-\mathrm{C}(12)-\mathrm{C}(13) \rightarrow$

$\mathrm{Cg} 3=\mathrm{C}(18)-\mathrm{C}(19)-\mathrm{C}(20)-\mathrm{C}(21)-\mathrm{C}(22)-\mathrm{C}(23) \rightarrow$ 
$\# 1: \mathrm{x},-\mathrm{y}, 1 / 2+\mathrm{z} ; \# 2: \mathrm{x},-\mathrm{y},-1 / 2+\mathrm{z}$

For 2:

$\mathrm{Cg} 1=\mathrm{C} 1-\mathrm{C} 2-\mathrm{C} 3-\mathrm{C} 4-\mathrm{C} 5-\mathrm{C} 6 \rightarrow$

$\mathrm{Cg} 2=\mathrm{C} 7-\mathrm{C} 8-\mathrm{C} 9-\mathrm{C} 10-\mathrm{C} 11-\mathrm{C} 12 \rightarrow$

$\mathrm{Cg} 3=\mathrm{C} 13-\mathrm{C} 14-\mathrm{C} 15-\mathrm{C} 16-\mathrm{C} 17-\mathrm{C} 18 \rightarrow$

\#1: -x, 1/2+y, 1/2-z; \#2: -x, 1-y, -z; \#3: -x, -1/2+y, 1/2-z

For 3:

$\mathrm{Cg} 1=\mathrm{C} 1-\mathrm{C} 2-\mathrm{C} 3-\mathrm{C} 4-\mathrm{C} 5-\mathrm{C} 6 \rightarrow$

$\mathrm{Cg} 2=\mathrm{C} 7-\mathrm{C} 8-\mathrm{C} 9-\mathrm{C} 10-\mathrm{C} 11-\mathrm{C} 12 \rightarrow$

$\mathrm{Cg} 3=\mathrm{C} 13-\mathrm{C} 14-\mathrm{C} 15-\mathrm{C} 16-\mathrm{C} 17-\mathrm{C} 18 \rightarrow$

$\# 1=2-x, 1 / 2+y, 3 / 2-z ; \# 2=2-x,-1 / 2+y, 3 / 2-z ; \# 3=2-x, 3-y, 2-z$.

\begin{tabular}{|l|l|l|l|l|l|l|}
\hline $\mathrm{Cg}(\mathrm{I}) \cdots \mathrm{Cg}(\mathrm{J})$ & $\mathrm{Cg} \cdots \mathrm{Cg}$ & Alpha & Beta & Gamma & CgI_Perp & CgJ_Perp \\
\hline Compound 1 \\
\hline $\mathrm{Cg} 2 \cdots \mathrm{Cg} 1 \# 1$ & $5.1214(2)$ & 83 & 14.0 & 79.0 & 0.9746 & 4.9703 \\
\hline $\mathrm{Cg} 2 \cdots \mathrm{Cg} 2 \# 2$ & $5.1533(2)$ & 17 & 33.4 & 36.0 & 4.1666 & -4.3042 \\
\hline $\mathrm{Cg} 2 \cdots \mathrm{Cg} 2 \# 1$ & $5.1533(2)$ & 17 & 36.0 & 33.4 & -4.3042 & 4.1666 \\
\hline $\mathrm{Cg} 2 \cdots \mathrm{Cg} 3 \# 2$ & $5.2202(2)$ & 84 & 23.5 & 70.6 & -1.7324 & -4.7881 \\
\hline $\mathrm{Compound} 2$ \\
\hline $\mathrm{Cg} 1 \cdots \mathrm{Cg} 2 \# 1$ & $5.2408(4)$ & 53 & 41.4 & 46.8 & -3.5900 & -3.9310 \\
\hline $\mathrm{Cg} 1 \cdots \mathrm{Cg} 3 \# 1$ & $4.0706(3)$ & 8 & 29.6 & 24.2 & -3.7128 & -3.5405 \\
\hline $\mathrm{Cg} 1 \cdots \mathrm{Cg} 3 \# 2$ & $4.7294(3)$ & 27 & 59.3 & 36.2 & 3.8162 & 2.4144 \\
\hline $\mathrm{Cg} 2 \cdots \mathrm{Cg} 1 \# 3$ & $5.2408(4)$ & 53 & 46.8 & 41.4 & -3.9310 & -3.5900 \\
\hline $\mathrm{Cg} 2 \cdots \mathrm{Cg} 2 \# 2$ & $4.7312(3)$ & 0 & 31.2 & 31.2 & 4.0458 & 4.0458 \\
\hline $\mathrm{Cg} 2 \cdots \mathrm{Cg} 3 \# 2$ & $5.0238(4)$ & 51 & 33.7 & 44.0 & 3.6152 & 4.1816 \\
\hline $\mathrm{Cg} 3 \cdots \mathrm{Cg} 1 \# 3$ & $4.0706(3)$ & 8 & 24.2 & 29.6 & -3.5405 & -3.7128 \\
\hline $\mathrm{Cg} 3 \cdots \mathrm{Cg} 1 \# 2$ & $4.7294(3)$ & 27 & 36.2 & 59.3 & 2.4144 & 3.8162 \\
\hline $\mathrm{Cg} 3 \cdots \mathrm{Cg} 2 \# 2$ & $5.0238(4)$ & 51 & 44.0 & 33.7 & 4.1816 & 3.6152 \\
\hline $\mathrm{Compound} 3$ & \multicolumn{5}{|l|}{$\mathbf{l}$} \\
\hline $\mathrm{Cg} 1 \cdots \mathrm{Cg} 2 \# 1$ & $5.451(8)$ & $52.8(6)$ & 26.6 & 56.3 & $-3.022(5)$ & $4.874(6)$ \\
\hline $\mathrm{Cg} 1 \cdots \mathrm{Cg} 3 \# 1$ & $4.405(8)$ & $18.9(7)$ & 25.3 & 23.4 & $4.043(6)$ & $3.984(5)$ \\
\hline $\mathrm{Cg} 2 \cdots \mathrm{Cg} 1 \# 2$ & $5.452(8)$ & $52.8(6)$ & 56.3 & 26.6 & $4.875(6)$ & $-3.023(5)$ \\
\hline $\mathrm{Cg} 2 \cdots \mathrm{Cg} 2 \# 3$ & $5.581(8)$ & $0.0(6)$ & 40.8 & 40.8 & $4.224(5)$ & $4.224(5)$ \\
\hline $\mathrm{Cg} 2 \cdots \mathrm{Cg} 3 \# 3$ & $5.500(8)$ & $65.6(6)$ & 40.1 & 38.1 & $-4.330(6)$ & $4.207(5)$ \\
\hline $\mathrm{Cg} 3 \cdots \mathrm{Cg} 1 \# 2$ & $4.405(8)$ & $18.9(7)$ & 23.4 & 25.3 & $3.984(5)$ & $4.043(6)$ \\
\hline $\mathrm{Cg} 3 \cdots \mathrm{Cg} 1 \# 3$ & $5.371(8)$ & $45.5(7)$ & 39.8 & 76.2 & $-1.277(5)$ & $-4.124(6)$ \\
\hline $\mathrm{Cg} 3 \cdots \mathrm{Cg} 2 \# 3$ & $5.501(8)$ & $65.6(6)$ & 38.1 & 40.1 & $4.207(5)$ & $-4.330(6)$ \\
\hline
\end{tabular}

Table S3. Analysis of $\mathrm{X}-\mathrm{H} \cdots \mathrm{Cg}(\mathrm{Pi}$-Ring) Interactions $(\mathrm{H} \cdots \mathrm{Cg}<3.0$ Ang. - Gamma $<$ 30.0 Deg)

- $\mathrm{Cg}(\mathrm{J})=$ Center of gravity of ring $\mathrm{J}$ (Plane number above)

- H-Perp $=$ Perpendicular distance of $\mathrm{H}$ to ring plane $\mathrm{J}$

- Gamma = Angle between $\mathrm{Cg}-\mathrm{H}$ vector and ring J normal 
- $\mathrm{X}-\mathrm{H} \cdots \mathrm{Cg}=\mathrm{X}-\mathrm{H}-\mathrm{Cg}$ angle (degrees)

- $\mathrm{X} \cdots \mathrm{Cg}=$ Distance of $\mathrm{X}$ to $\mathrm{Cg}$ (Angstrom)

- X-H, Pi = Angle of the X-H bond with the Pi-plane (i.e.' Perpendicular $=90$ degrees, Parallel $=0$ degrees $)$

\begin{tabular}{|c|c|c|c|c|c|c|}
\hline $\mathrm{X}-\mathrm{H} \cdots \mathrm{Cg}(\mathrm{J})$ & $\mathrm{H} \cdots \mathrm{Cg}$ & H-Perp & Gamma & $\mathrm{X}-\mathrm{H} \cdots \mathrm{Cg}$ & $\mathrm{X} \cdots \mathrm{Cg}$ & $\mathrm{X}-\mathrm{H} \cdots \mathrm{Pi}$ \\
\hline \multicolumn{7}{|l|}{ Compound 1} \\
\hline $\mathrm{C}(4)-\mathrm{H}(4 \mathrm{~A}) \cdots \mathrm{Cg}(2) \# 1$ & 2.92 & 2.80 & 16.49 & 156 & $3.7898(1)$ & 71 \\
\hline \multicolumn{7}{|l|}{ Compound $\mathbf{2}$} \\
\hline $\mathrm{C} 20-\mathrm{H} 20 \mathrm{~B} \cdots \mathrm{Cg} 2 \# 1$ & 2.82 & -2.80 & 6.78 & 142 & $3.6246(3)$ & 50 \\
\hline \multicolumn{7}{|l|}{ Compound $\mathbf{3}$} \\
\hline $\mathrm{C} 23-\mathrm{N} 1 \cdots \mathrm{Cg} 1 \# 1$ & $3.92(3)$ & 3.820 & 12.99 & $108.7(17)$ & $4.61(3)$ & 30.95 \\
\hline
\end{tabular}

Symmetry transformations used to generate equivalent atoms: For 1: \#1 $=\mathrm{x},-\mathrm{y}$,

$-1 / 2+z$. For 2: \#1: $-\mathrm{x}, 1 / 2+\mathrm{y}, 1 / 2-\mathrm{z}$. For 3: \#1: 1+x, 5/2-y, 1/2+z

Table S4. Hydrogen bonds in compounds 1-3.

\begin{tabular}{|c|c|c|c|c|}
\hline Donor-H $\cdots$ Acceptor & D-H & $\mathrm{H} \cdots \mathrm{A}$ & $\mathrm{D} \cdots \mathrm{A}$ & $\mathrm{D}-\mathrm{H} \cdots \mathrm{A}$ \\
\hline \multicolumn{5}{|l|}{ Compound 1} \\
\hline O1W-H1W $\cdots$ O1W\#1 & 0.85 & 1.46 & $1.8693(1)$ & 105 \\
\hline $\mathrm{O} 1 \mathrm{~W}-\mathrm{H} 2 \mathrm{~W} \cdots \mathrm{O} 1 \mathrm{~W} \# 1$ & 0.85 & 1.43 & $1.8693(1)$ & 107 \\
\hline C7-H7A $\cdots \mathrm{O} 1$ & 0.93 & 2.48 & $2.7910(1)$ & 100 \\
\hline \multicolumn{5}{|l|}{ Compound $\mathbf{2}$} \\
\hline O1W-H1W $\cdots$ O2\#1 & 0.85 & 1.98 & $2.7361(2)$ & 148 \\
\hline O1W-H1W $\cdots$ O3\#2 & 0.85 & 2.54 & $3.1405(2)$ & 128 \\
\hline $\mathrm{O} 1 \mathrm{~W}-\mathrm{H} 2 \mathrm{~W} \cdots \mathrm{O} 2 \mathrm{~W} \# 3$ & 0.85 & 1.82 & $2.6555(2)$ & 167 \\
\hline $\mathrm{O} 2 \mathrm{~W}-\mathrm{H} 3 \mathrm{~W} \cdots \mathrm{O} 3 \mathrm{~W}$ & 0.85 & 2.52 & $3.1351(2)$ & 130 \\
\hline O3W-H6W $\cdots \mathrm{O} 1$ & 0.85 & 2.08 & $2.9289(2)$ & 174 \\
\hline C16-H16A $\cdots$ O6\#4 & 0.93 & 2.57 & $3.4697(3)$ & 163 \\
\hline \multicolumn{5}{|l|}{ Compound $\mathbf{3}$} \\
\hline $\mathrm{O} 1 \mathrm{~W}-\mathrm{H} 1 \mathrm{~W} \cdots \mathrm{N} 1$ & 0.85 & 1.96 & $2.77(2)$ & 157 \\
\hline $\mathrm{O} 1 \mathrm{~W}-\mathrm{H} 2 \mathrm{~W} \cdots \mathrm{O} 1 \# 1$ & 0.85 & 1.86 & $2.676(13)$ & 162 \\
\hline $\mathrm{C} 16-\mathrm{H} 16 \mathrm{~A} \cdots \mathrm{O} 6$ & 0.93 & 2.55 & $3.455(15)$ & 163 \\
\hline $\mathrm{C} 18-\mathrm{H} 18 \mathrm{~A} \cdots \mathrm{O} 3$ & 0.93 & 2.42 & $2.741(14)$ & 100 \\
\hline $\mathrm{C} 24-\mathrm{H} 24 \mathrm{~B} \cdots \mathrm{N} 1 \# 2$ & 0.96 & 2.19 & $3.02(4)$ & 145 \\
\hline $\mathrm{C} 24-\mathrm{H} 24 \mathrm{C} \cdots \mathrm{O} 2 \# 3$ & 0.96 & 2.59 & $3.29(2)$ & 130 \\
\hline
\end{tabular}

Symmetry transformations used to generate equivalent atoms: For 1: \#1: $-\mathrm{x}, \mathrm{y}, 1 / 2-\mathrm{z}$. For 2: \#1: 1-x, -1/2+y, 3/2-z; \#2: -x, -1/2+y, 1/2-z; \#3: x, 1/2-y, 1/2+z; \#4:-1+x, y, -1+z. For 3: $\# 1$ = 2-x, -1/2+y, 3/2-z; \#2 = 3-x, 3-y, 2-z; \#3 = 2-x, 1/2+y, 3/2-z. 


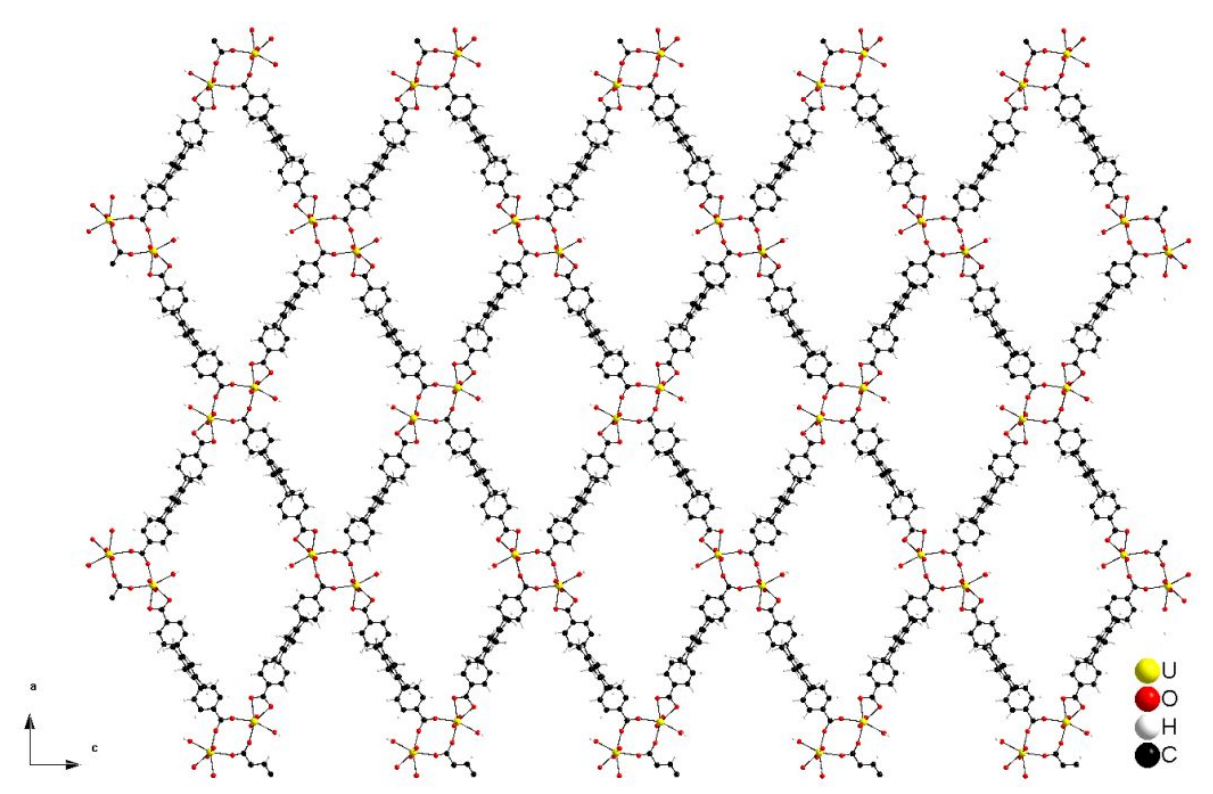

Figure S5. One framework in compound 1 viewing along $b$-direction.



(a)

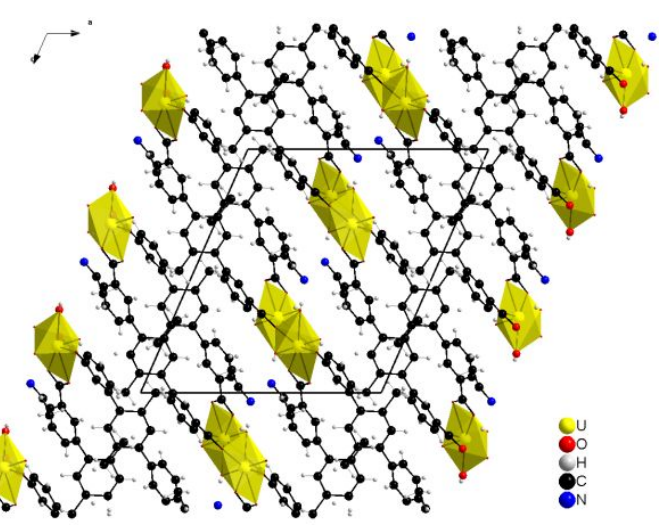

(b)

Figure S6. Two-dimensional layer in $b c$-plane (a) and three-dimensional supramolecular structure viewing along $b$-direction (b) of compound 3. The $\mathrm{UO}_{7}$ polyhedrons are shaded in yellow.

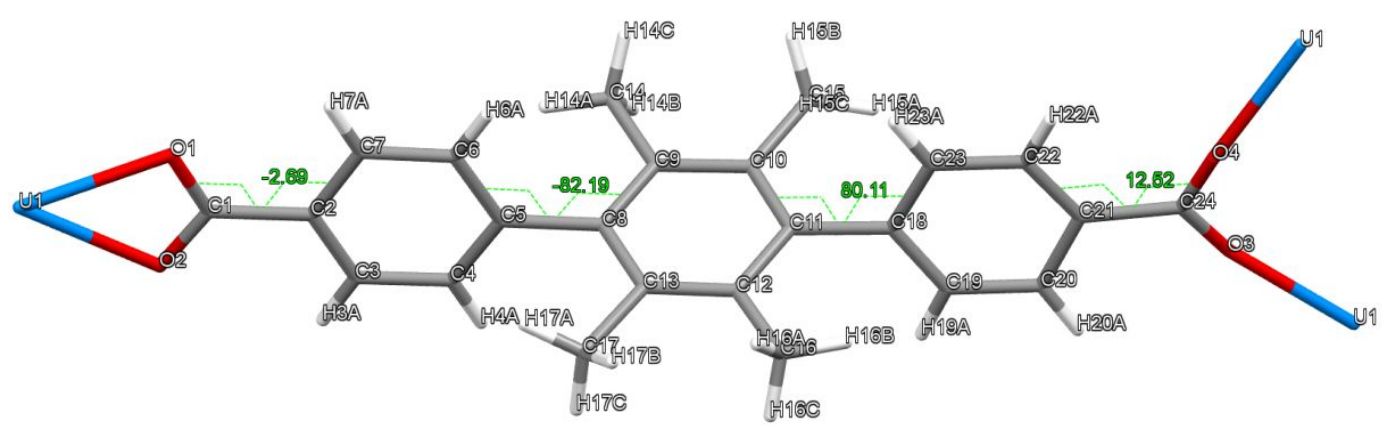

(a) 


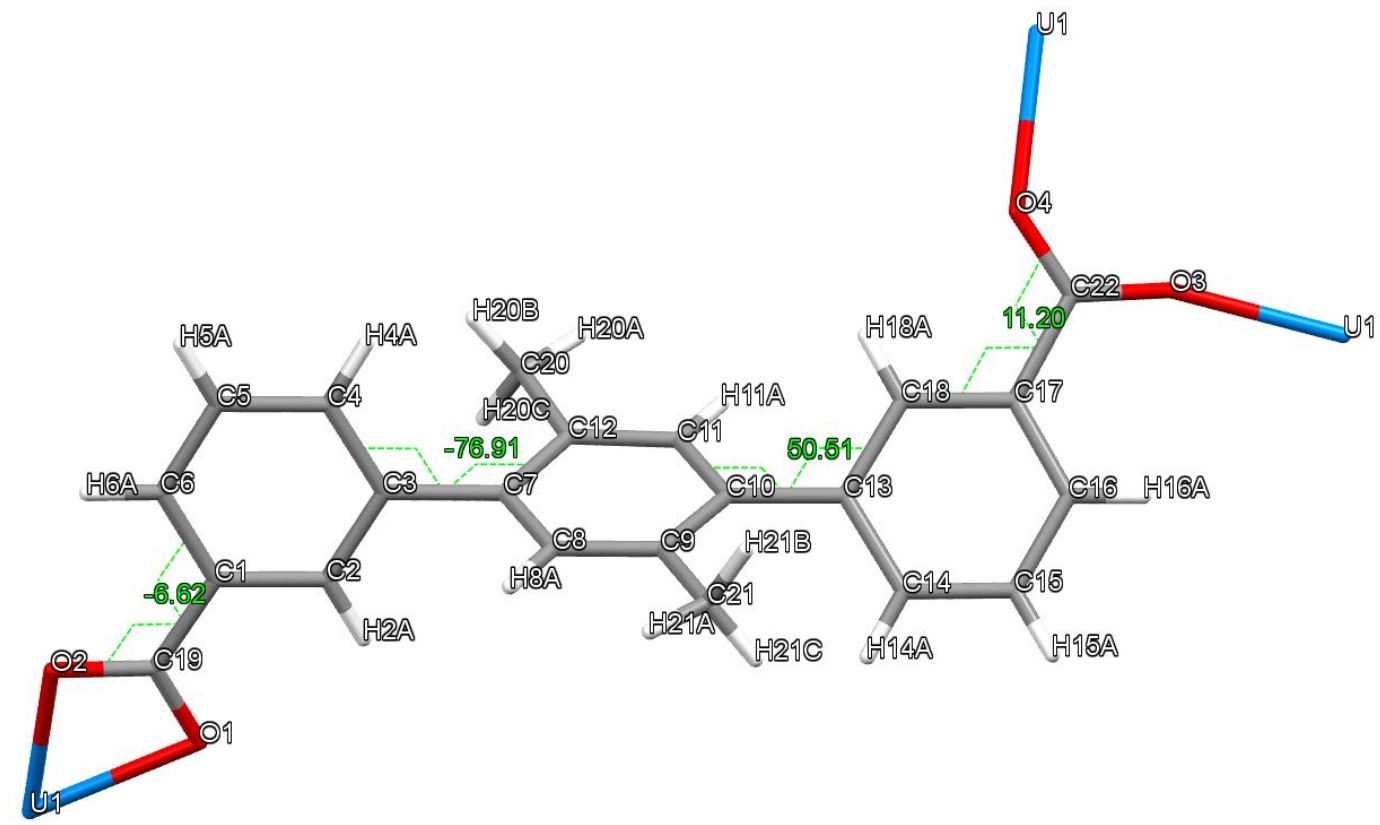

(b)

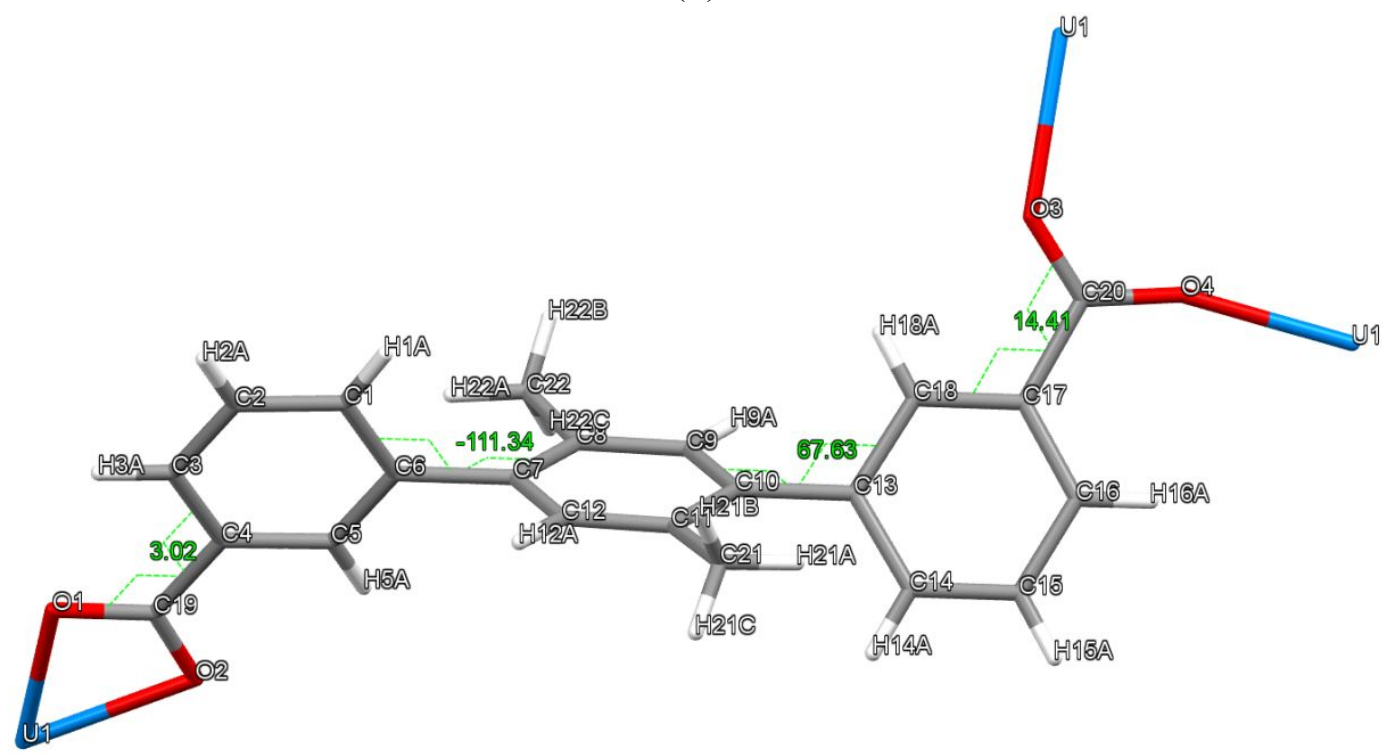

(c)

Figure S7. Torsion angles between neighboring carboxylic and benzene rings in the carboxylate ligands of compounds 1-3. 


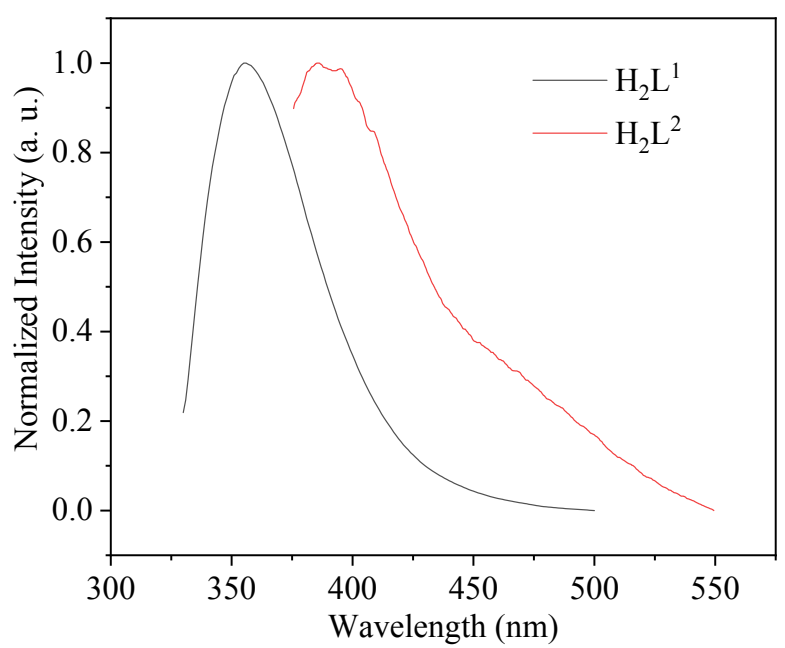

Figure S8. Normalized emission spectra of the two carboxylic ligands.

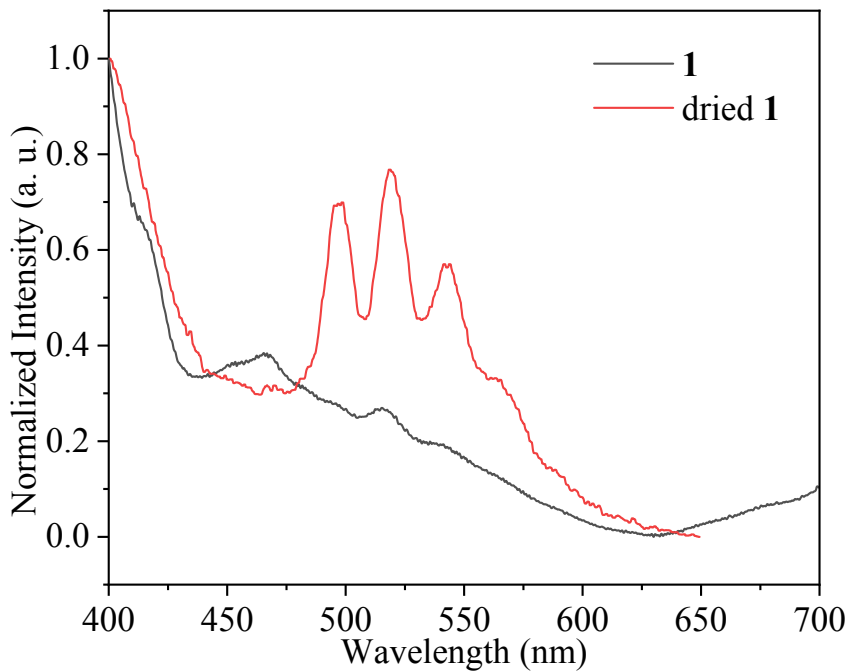

Figure S9. Normalized emission spectra of compound $\mathbf{1}$ and dried $\mathbf{1}$.

Dried 1 was obtained by immersing the fresh single crystals of compound 1 in methanol for three days, during which the methanol was changed every six hours. Then the crystals were dried in a vacuum oven at $60{ }^{\circ} \mathrm{C}$ for 24 hours. 